INPLASY

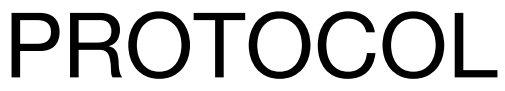

To cite: Liu et al. Effectiveness and safety of Yufengningxin for treating coronary heart disease angina: A protocol for a systematic review and metaanalysis. Inplasy protocol 2020110040. doi:

10.37766/inplasy2020.11.0040

Received: 10 November 2020

Published: 10 November 2020

Corresponding author: Chao Liu

liuchao0725@bucm.edu.cn

Author Affiliation:

Department of Cardiology, Guang'anmen Hospital, China academy of Chinese medical Sciences, Beijing, China

Support: "Qihuang scholars" Project.

Review Stage at time of this submission: Preliminary searches.

Conflicts of interest: None.

\section{Effectiveness and safety of Yufengningxin for treating coronary heart disease angina: A protocol for a systematic review and meta-analysis}

\author{
Liu, C1; Bai, J2; Liu, LC3; Gao, JL4; Wang, J5.
}

Review question / Objective: Yufengingxin (YFNX) is a commonly used Chinese patent medicine for the treatment of coronary heart disease (CHD) angina. The objective of this protocol is to evaluate the effectiveness and safety of YFNX in CHD angina treatment.

Condition being studied: Coronary heart disease (CHD) angina, a common cardiovascular disease, is caused by coronary artery stenosis or spasm, which leads to myocardial ischemia and hypoxia. At present, 11396,000 people in China suffer from CHD, and the mortality rate of urban/rural residents is 115.32 per 100,000 and 122.04 per 100,000 , respectively. CHD seriously affects people's quality of life and causes a huge economic burden on the society. Nitrates are the most commonly used drugs for relieving angina pectoris. However, the use of nitrates has been found to have some side effects in clinical practice, such as dizziness, blushing, nausea, and loss of appetite. Therefore, novel strategies for alleviating symptoms of angina need to be explored, especially with no obvious side effects. Yufengningxin (YFNX) is extracted and processed from Radix Puerariae, which is a commonly used Chinese patent medicine for the treatment of CHD. However, there is still no strong evidence on the effectiveness and safety of YFNX for treating CHD angina.

INPLASY registration number: This protocol was registered with the International Platform of Registered Systematic Review and Meta-Analysis Protocols (INPLASY) on 10 November 2020 and was last updated on 10 November 2020 (registration number INPLASY2020110040).

\section{INTRODUCTION}

Review question / Objective: Yufengingxin (YFNX) is a commonly used Chinese patent medicine for the treatment of coronary heart disease (CHD) angina. The objective of this protocol is to evaluate the effectiveness and safety of YFNX in CHD angina treatment. 
Condition being studied: Coronary heart disease (CHD) angina, a common cardiovascular disease, is caused by coronary artery stenosis or spasm, which leads to myocardial ischemia and hypoxia. At present, 11396,000 people in China suffer from CHD, and the mortality rate of urban/rural residents is 115.32 per 100,000 and 122.04 per 100,000 , respectively. CHD seriously affects people's quality of life and causes a huge economic burden on the society. Nitrates are the most commonly used drugs for relieving angina pectoris. However, the use of nitrates has been found to have some side effects in clinical practice, such as dizziness, blushing, nausea, and loss of appetite. Therefore, novel strategies for alleviating symptoms of angina need to be explored, especially with no obvious side effects. Yufengningxin (YFNX) is extracted and processed from Radix Puerariae, which is a commonly used Chinese patent medicine for the treatment of CHD. However, there is still no strong evidence on the effectiveness and safety of YFNX for treating CHD angina.

\section{METHODS}

Participant or population: Patients with CHD angina.

Intervention: The intervention group took YFNX combined with conventional therapy. We will include various dosage forms of YFNX, including tablets, dripping pills, capsules, granules and so on.

Comparator: The control group used conventional therapy, such as aspirin, clopidogrel, $\beta$-blockers, statins, reninangiotensin-aldosterone system blockers, calcium channel blockers, nitrates.

Study designs to be included: All relevant randomized controlled trials (RCTs) regarding YFNX for the treatment of CHD angina will be included, and meanwhile no limitation of the language and the type of publication.

Eligibility criteria: Patients with CHD angina, including stable angina pectoris (SAP) and unstable angina (UA), according to the World Health Organization (WHO) diagnostic criteria for CHD. The gender, age, ethnic and region will not be considered.

Information sources: We will conduct a systematic search of the relevant databases, including PubMed, Cochrane Library, EMBASE, Chinese National Knowledge Infrastructure (CNKI), Wanfang database, Chinese Scientific Journal database (VIP) and Chinese biomedical literature database (CBM), from their inception to November 1, 2020. The English search terms include YFNX, CHD, angina pectoris and randomized controlled trials. We will also manually retrieve grey literature and clinical trial registers, such as WHO International Clinical Trials Registry Platform (ICTRP), the Chinese Clinical Trial Register (ChiCTR) and the Clinical Trials.

Main outcome(s): The frequency and duration of angina pectoris attacks.

Quality assessment / Risk of bias analysis: The Cochrane risk of bias tool will be used to assess the risk of bias in each included studies by two independent authors. The main items include random sequence generation, allocation concealment, blinding of subjects and test personnel, blinding of outcome evaluator, incomplete outcome report, selective report study results, and other biases.

Strategy of data synthesis: The RevMan $\mathbf{5 . 3}$ software (Cochrane Collaboration, Copenhagen, Denmark) will be used for data synthesis.

Subgroup analysis: If the included studies have significant heterogeneity and more than 10 trials, we will perform subgroup analysis based on the patient's age, type of angina pectoris, dosage form of YFNX, adverse events, and so on.

Sensibility analysis: We will perform a sensitivity analysis by eliminating each study individually or changing the category of the effect model to ensure the robustness of analytical conclusions. 
Country(ies) involved: China.

Keywords: Yufengningxin, coronary heart disease angina, protocol, systematic review, meta-analysis.

Contributions of each author:

Author 1 - Chao Liu.

Author 2 - Jing Bai.

Author 3 - Lanchun Liuo.

Author 4 - Jialiang Gao.

Author 5 - Jie Wang. 